\title{
Phronesis
}

\section{Les acteurs de l'éducation et de la formation face aux défis des savoirs}

\author{
Enjeux de recherche, enjeux de pratique
}

Philippe Maubant et Stéphane Martineau

Volume 1, numéro 2, avril 2012

URI : https://id.erudit.org/iderudit/1009055ar

DOI : https://doi.org/10.7202/1009055ar

Aller au sommaire du numéro

Éditeur(s)

Institut de recherche sur les pratiques éducatives

ISSN

1925-4873 (numérique)

Découvrir la revue

Citer ce document

Maubant, P. \& Martineau, S. (2012). Les acteurs de l'éducation et de la

formation face aux défis des savoirs : enjeux de recherche, enjeux de pratique.

Phronesis, 1(2), 1-4. https://doi.org/10.7202/1009055ar d'utilisation que vous pouvez consulter en ligne.

https://apropos.erudit.org/fr/usagers/politique-dutilisation/ 


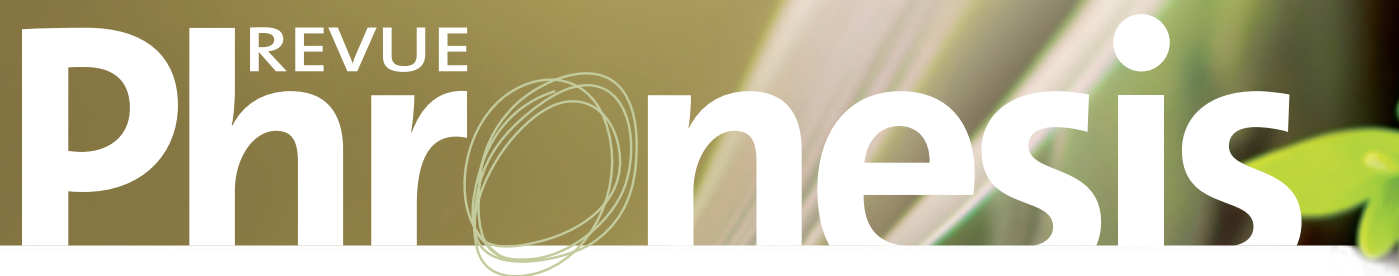

Institut de recherche sur les pratiques éducatives de l'Université de Sherbrooke

\section{LES ACTEURS DE L'ÉDUCATION ET DE LA FORMATION FACE AUX DÉFIS DES SAVOIRS}

Philippe Maubant $^{\star}$ et Stéphane Martineau ${ }^{\star *}$

${ }^{*}$ Institut de recherche sur les pratiques éducatives

Université de Sherbrooke

2500 boul de l'Université Sherbrooke, Québec, Canada, J1K 2R1

Philippe.Maubant@USherbrooke.ca

${ }^{*}$ Laboratoire du développement et d'insertion professionnelle en enseignement

Université du Québec à Trois-Rivières

C.P. 500 Trois-Rivières, Québec, Canada, G9A 5H7

stephane.martineau@uqtr.ca

ENJEUX DE RECHERCHE, ENJEUX DE PRATIQUE 
Dans ce contexte, les professionnels de léducation et de la formation sont directement interpelés. L’environnement éducatif dans lequel ils évoluent se transforme sans arrêt, ce qui les place devant l'injonction constante de devoir s’adapter, sans qu'ils aient nécessairement la possibilité de développer une pensée critique sur les valeurs et les finalités des dispositifs dans lesquels ils sont impliqués (Fabre, 2011). Ces acteurs de léducation et de la formation sont constamment invités à développer une posture réflexive sur les pratiques éducatives qu'ils conçoivent, planifient et mettent en œuvre. Mais disposent-ils des contextes, des situations et des temporalités propices à ce nécessaire et salutaire moratoire de laction éducative (Maradan \& Vivier, 2011) ? La communauté scientifique des chercheurs en sciences de léducation a répondu aux orientations de certains décideurs qui soutenaient un changement radical des politiques éducatives pour les mettre au service de la doxa néolibérale. Le plus souvent de manière critique, et en cela ils restent dans leur rôle, les scientifiques ont alerté la société et les décideurs sur certaines contradictions ou zones d’ombre des nouvelles politiques éducatives. Ils ont aussi contribué à évaluer limpact et les effets de modèles pédagogiques ou de pratiques cherchant à répondre ou à résoudre des problématiques tout autant scolaires que sociales, comme le décrochage des élèves du secondaire, par exemple. Les recherches en sciences de léducation, la discipline scientifique elle-même, sest donc positionnée au cœur d'une tension entre une visée pragmatique des recherches en sciences humaines et sociales et une ambition critique, autrement dit, entre d'une part une obligation d'impact sur les pratiques individuelles et collectives et, d'autre part, le nécessaire et indispensable devoir de mise en perspective critique des politiques et du fonctionnement des sociétés (Develay, 2001). Dans cette perspective, les financements publics des recherches en sciences humaines et sociales encouragent, ces dernières années, les programmes et travaux visant à modifier ou à transformer les pratiques professionnelles en ayant pour visée, in fine, l'accroissement de lefficacité des travailleurs, quel que soit le contexte professionnel. Les acteurs de léducation et de la formation néchappent pas à cette tendance et au discours croissant sur la nécessité d’améliorer la qualité des pratiques des formateurs, celles des enseignants et celles des différents intervenants éducatifs sont une réalité des politiques déducation et de formation. C’est en ce sens que la question de la professionnalisation des métiers relationnels, incluant les métiers de léducation et de la formation, autrement dit les métiers adressés à autrui (Piot, 2009), constitue un enjeu scientifique important et un défi social majeur.

Rappelons que cette problématique de la professionnalisation concerne tout autant létude des politiques et des dispositifs de formation, lanalyse de l'agir professionnel ou encore létude des situations formatives. Ce numéro 2 de la revue Phronesis propose d’interroger la question de lanalyse de l’agir professionnel dans les métiers de léducation et de la formation. Outre la question des différents types de savoirs mobilisés par ces professionnels, la question de leur rapport aux savoirs, la place des savoirs de l’expérience dans l'intervention éducative, lanalyse du «bricolage» que font les praticiens des différents savoirs qu'ils possèdent et maitrisent pour intervenir, etc. (Donnay \& Bru, 2002), le rôle des dispositifs de formation dans la construction des savoirs professionnels sont autant de regards que ce numéro propose de convoquer et de débattre.

Ce numéro fait suite à prolonge les réflexions amorcées lors de deux colloques. Il s'inscrit tout d’abord directement dans la suite du colloque que nous avons organisé en 2008, lors du 15e congrès de l'Association mondiale des sciences de léducation (AMSÉ) qui s’est tenu à Marrakech. Il poursuit également les échanges et les discussions tenus à loccasion d’un symposium - organisé dans le cadre des activités du réseau-observatoire international sur la professionnalisation dans les métiers relationnels et de l'interaction humaine - qui a eu lieu, cette fois, dans le cadre du 16e congrès de l'AMSÉ. Nous souhaitons ainsi non seulement prolonger et mais aussi élargir la réflexion sur la problématique des savoirs professionnels des acteurs impliqués dans léducation (qu'il s’agisse des enseignants du primaire, du secondaire et dupostsecondaire), dans la formation professionnelle, dans la formation des adultes, dans lensemble des métiers relationnels ayant une visée déducation ou de formation. Plus spécifiquement, ce numéro nous invite au dialogue entre chercheurs de plusieurs pays, entre chercheurs et décideurs, entre chercheurs et formateurs autour des questions suivantes : Qu’entend-on par savoirs professionnels? Quels en sont leurs fondements épistémologiques? Comment les identifier dans lanalyse du travail ? Comment les prendre en compte dans les situations de formation ? Sous quelles conditions peuvent-ils aider à labourer des situations d’apprentissage professionnel ? Quelles places ont-ils dans les dispositifs de formation professionnelle initiale et/ou continue ? L’objectif général de ce numéro est donc de mieux comprendre les défis que pose la question des savoirs professionnels pour les recherches sur l'agir professionnel dans les métiers de léducation et de la formation. 


\section{Références bibliographiques}

Bozio, A., Dauvergne, R.\& Fabre, B. (2012). Fiscalité et redistribution en France, 1997-2002. Rapport de l'Institut des politiques publiques. Téléaccessible à l’adresse suivante : http://www.ipp.eu

Develay, M. (2001). Propos sur les sciences de l'éducation. Réflexions épistémologiques. Paris : ESF.

Donnay, J.\& Bru, M. (dir.) (2002). Recherches, pratiques et savoirs en éducation. Paris : PUF.

Fabre, M. (2011). Éduquer pour un monde problématique. La carte et la boussole. Paris : PUF.

Freitag, M. (2008). L'impasse de la globalisation. Une histoire sociologique et philosophique du capitalisme. Montréal : Écosociété.

Lamarche, T. (dir.) (2006). Capitalisme et éducation. Paris : Nouveaux Regards.

Laroche, J. (2012). La brutalisation du monde. Du retrait des États à la décivilisation. Montréal : Liber.

Maranda, M.-F.\& Viviers, S. (dir.). (2011). Lécole en souffrance. Psychodynamique du travail en milieu scolaire. Québec : PUL.

Piot, T. (2009). Quels indicateurs pour mesurer le développement professionnel dans les métiers adressés à autrui ? Questions vives (5), p. 259-275.

Stiglitz, J. (2010). Le triomphe de la cupidité. Paris : LLL.

Tardif, M., Lessard, C.\& Gauthier, C. (dir.) (1998). Formation des maîtres et contextes sociaux. Paris : PUF. 